\title{
Targeting cell cycle protein in gastric cancer with CDC20siRNA and anticancer drugs (doxorubicin and quercetin) co-loaded cationic PEGylated nanoniosomes
}

\author{
Mahdie Hemati ${ }^{1,2}$ \\ Fateme Haghiralsadat ${ }^{2,3}$ \\ Farzaneh Jafary' \\ Seyedmohammad \\ Moosavizadeh ${ }^{4}$ \\ Ali Moradi ${ }^{1}$
}

'Department of Clinical Biochemistry, Faculty of Medicine, Shahid Sadoughi University of Medical Sciences, Yazd, Iran;

${ }^{2}$ Medical Nanotechnology and Tissue Engineering Research Center, Yazd Reproductive Sciences Institute, Shahid Sadoughi University of Medical Sciences, Yazd, Iran, ${ }^{3}$ Department of Advanced Medical Sciences and Technologies, School of Paramedicine, Shahid Sadoughi University of Medical Sciences, Yazd, Iran; ${ }^{4}$ Department of Laboratory Sciences, School of Paramedicine, Shahid Sadoughi University of Medical Sciences, Yazd, Iran

Correspondence: Ali Moradi

Department of Clinical Biochemistry, Faculty of Medicine, Shahid Sadoughi

University of Medical Sciences and Health

Services, Yazd, Iran

Tel +989126706056

$\mathrm{Fax}+988915173149$

Email moradi20018@gmail.com
This article was published in the following Dove Press journal:

International Journal of Nanomedicine

Background and purpose: In a past study, we developed and optimized a novel cationic PEGylated niosome containing anticancer drugs (doxorubicin or quercetin) and siRNA. This study intended to evaluate the anti-tumor effects of the combination therapy to target both the proteins and genes responsible for the development of gastric cancer. CDC20, known as an oncogene, is a good potential therapeutic candidate for gastric cancer.

Methods: In order to increase the loading capacity of siRNA and achieve appropriate physical properties, we optimized the cationic PEGylated niosome in terms of the amount of the cationic lipids. Drugs (doxorubicin and quercetin) and CDC20siRNA were loaded into the co-delivery system, and physical characteristics, thermosensitive controlled-release, gene silencing efficiency, and apoptosis rate were determined.

Results: The results showed that the designed co-delivery system for the drugs and gene silencer had an appropriate size and a high positive charge for loading siRNA, and also showed a thermosensitive drug release behavior, which successfully silenced the CDC20 expression when compared with the single delivery of siRNA or the drug. Moreover, the codelivery of drugs and CDC20siRNA exhibited a highly inhibitory property for the cell growth of gastric cancer cells.

Conclusion: It seems that the novel cationic PEGylated niosomes co-loaded with anticancer drug and CDC20siRNA has a promising application for the treatment of gastric cancer.

Keywords: niosome, siRNA, CDC20, doxorubicin, quercetin

\section{Introduction}

Gastric cancer is one of the most common cancers with a generally poor prognosis. ${ }^{1}$ Chemotherapy is currently the main cancer treatment option for the therapy of various types of cancers. However, the use of chemotherapy has raised concerns regarding the adverse effects on the healthy tissues and normal cells. Therefore, drug delivery to cancerous tissues is considered a major necessity. The most important aim of this study was to overcome the two main obstacles existing in the treatment of cancer, the first is to reduce the side effects, and the second is to conquer the drug resistance. In order to decrease the side effects and increase the efficacy of drugs and the delivery rate to a particular target, nanocarriers have been showing promise for the delivery of therapeutics in recent years. ${ }^{2,3}$ Nano-niosomes are nonionic surfactant vesicles, which have been used to deliver various hydrophobic and hydrophilic drugs, genes, hormones, 
antigens, and peptides. The targeted delivery and controlled drug release can decrease the adverse effects of chemotherapeutic agents and elevate the efficiency of the anti-tumor drug. Chemotherapy failure results from the development of the resistance to anti-cancer components. Therefore, the delivery of the different types of medications to tumor tissues through a multidrug delivery system can have synergistic anti-proliferative effects. Cell division cycle 20 homolog (CDC20) is anaphase-promoting complex (APC) activator and a regulatory protein that plays a vital role in the cell-cycle checkpoint. ${ }^{4}$ The overexpression of $\mathrm{CDC} 20$ has been shown to promote colorectal, ${ }^{5}$ pancreatic, ${ }^{6}$ non-small cell lung, ${ }^{7}$ and gastric cancers. ${ }^{8}$ Thus, the knock-down of the expression of CDC20 by the delivery of siRNA against CDC20 may have a great impact on the inhibition of gastric cancer cells. These results suggest that CDC20, as a key cell cycle regulator, has a potential therapeutic target for gastric cancer. Due to the divergence of cancer cell metabolism, the simultaneous administration of siRNA and chemotherapeutic agents such as doxorubicin (DOX) can lead to the highest therapeutic outcome. Quercetin (QC) as herbal medicine and chemosensitizer indicates anti-inflammatory and anticancer properties. ${ }^{9}$ However, the underlying mechanisms and its role in the suppression of tumor cells remains unclear. The effect of DOX and QC on the expression of CDC20 has not been so far investigated. In this study, we compared the efficacy of the free and single forms of siRNA and antiproliferative drugs (DOX and QC) with the niosome-encapsulated forms of the drugs and siRNA when applied alone or in combination with each other to suppress the CDC20 expression. We hypothesized that the combination of chemotherapeutic compounds and siRNA delivery could significantly diminish the expression of $\mathrm{CDC} 20$, and therefore, enhance the therapeutic efficacy of gastric cancer.

\section{Materials and methods}

Cholesterol and DOTAP (1, 2-dioleoyl-3-trimethylammonium-propane) were supplied by Sigma-Aldrich (St. Louis, MO, USA) and Avanti Polar Lipids (AL, USA), respectively. Tween-60 was purchased from DaeJung Chemicals $\&$ Metals (SouthKorea). DPPC (1, 2-dipalmitoyl-sn-glycero-3-phosphocholinephospholipid) and DSPE-PEG2000 (distearoyl phosphoethanolamine-polyethylene glycol) was obtained from Lipoid GmbH (Ludwigshafen, Germany). Doxorubicin as hydrochloride (DOX) and QC (purity $>95 \%$ ) were obtained from Ebewe Pharma (Austria) and Sigma-Aldrich (St Louis, MO, USA), respectively. The sequences of siRNA targeted the CDC20 mRNA were: sense 5'-GGGAAUAUAUAUCCUCUGUTT-3', antisense 5-ACAGAGGaUAUAUAUUCCCTT-3'. The sequences of siRNA were synthesized by Eurofins Genomics Ebersberg. CDC20 rabbit polyclonal antibody, actin mouse monoclonal antibody and Annexin V-FITC/PI apoptosis detection kit were supplied from Abcam (Abcam, Cambridge, UK). The other chemicals and solvents were of the analytical degree and used without further purification unless specified.

\section{Preparation of drug delivery system}

To achieve a cationic formulation for the dual drug/gene delivery, the optimization experiments for DOTAP contents were performed. A series of cationic PEGylated niosomes with various molar ratios of DOTAP $(0,10$, $20,25,30$ ) was synthesized by a thin-film hydration method, as described previously, ${ }^{10}$ and the dosage of both drugs, DOX and QC, was $0.5 \mathrm{mg} \cdot \mathrm{mL}^{-1}$.

\section{Determination of physical characteristics}

The particle size distribution, polydispersity index (PDI), and zeta potential of the obtained niosomes were evaluated by dynamic light scattering (DLS) technique using a particle size analyzer and ZetaPALS zeta potential Brookhaven Corp Instruments (Holtsville, NY USA). All measurements were performed three times, and their mean amounts were recorded.

\section{Morphological studies}

The shape and the surface morphology of the nanoniosomes were determined using images obtained from an atomic force microscope (AFM) (Nanowizard II; JPK instruments; Germany). The nanoniosome formulations were diluted to 1:1000 with deionized water and sonicated for about 20 mins in an ultrasonic agitator (E-Chrom Tech Co, Taiwan). The preparations were then deposited onto a mica sheet and observed under the AFM. In addition, the bilayer structure of the nanocarriers and its spherical shape were studied by Cryo-TEM (FEI Tecnai 20, type Sphera, OR, USA) at $200 \mathrm{kV}$.

\section{Differential scanning calorimetry (DSC)}

The phase transition temperature of niosomes was conducted using a DSC SDT Q600 (TA Instruments, New Castle, DE, USA), analyze the thermosensitivity of niosomes with $10^{\circ} \mathrm{C} / \mathrm{min}$ heating rate and a temperature range of $25-80^{\circ} \mathrm{C}$. 


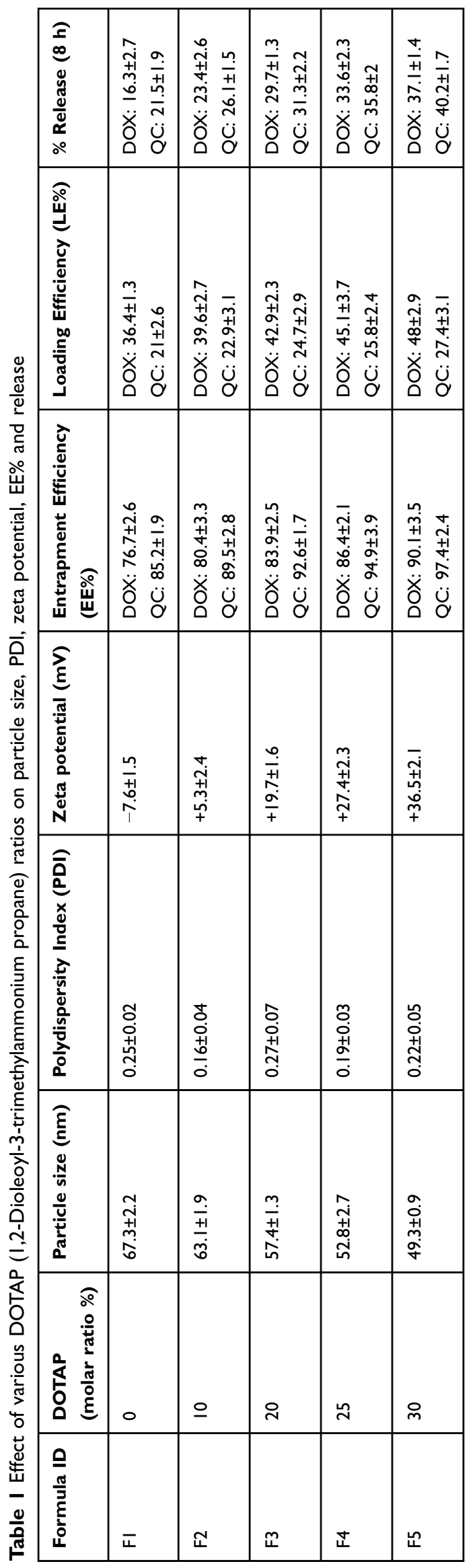

Determination of entrapment efficiency (EE\%) and loading efficiency (LE\%) of the drugs

The entrapment efficiency for DOX and QC were measured by a UV-Vis spectrophotometer (Perkin Elmer Germany) at $481 \mathrm{~nm}$ and $380 \mathrm{~nm}$, respectively. The entrapment efficiency for the mentioned drugs was calculated based on a standard curve and calculated by the following equation:

$$
\begin{array}{r}
\mathrm{EE} \%=\text { drug concentrationin niosome }\left(\mathrm{mg} \cdot \mathrm{ml}^{-1}\right) / \\
\text { Total drug }\left(\mathrm{mg} \cdot \mathrm{ml}^{-1}\right) \times 100
\end{array}
$$

The loading efficiency of DOX and QC were determined by the UV-Vis spectrophotometer (Perkin Elmer Germany) at $481 \mathrm{~nm}$ and $380 \mathrm{~nm}$ respectively using the following equation:

$$
\begin{array}{r}
\% \mathrm{LE}=\text { mass of drug in niosome }(\mathrm{mg}) / \\
\text { mass of niosome }(\mathrm{mg}) \times 100
\end{array}
$$

\section{In vitro drug release assay}

About $500 \mu \mathrm{L}$ of the niosome-loaded drugs were transferred into a $12 \mathrm{kDa}$ cut-off dialysis tube and incubated in $10 \mathrm{~mL}$ of PBS with a shaker shaken at $70 \mathrm{rpm}$ for $96 \mathrm{~h}$ in $\mathrm{pH} 7.4$ at $37^{\circ} \mathrm{C}$ and $42^{\circ} \mathrm{C}$ temperature (mimics the tumor tissue conditions). The medium of samples was drawn off at different times and substituted with the same volume of a fresh medium. Samples were measured by UV-Vis spectrophotometry at $481 \mathrm{~nm}$ and $380 \mathrm{~nm}$ for DOX and QC, respectively.

\section{In vitro evaluation of cellular uptake}

To evaluate the intracellular localization of the delivered Nio-DOX (Doxorubicin-loaded niosome), Nio-QC (Quercetin-loaded niosome), and Nio-FAM-labeled siRNA, as well as the effect of increasing concentration of DOTAP on enhanced penetration of cationic PEGylated nanoniosomes into the gastric cancer cells, DOX (red fluorescence), QC, and FAM-labeled siRNA (green fluorescence), were used to detect the cellular uptake. AGS, a cell line belonging to gastric cancer, was supplied by the Pasteur Institute (Tehran, Iran), seeded in a 6-well plate at $1.5 \times 10^{5}$ cells/well. Then, the cells were treated with NioDOX, Nio-QC, and Nio-FAM-labeled siRNA at both formulations namely F2 and F5 (Table 1) for 8 hrs. The cells were then rinsed twice with PBS (pH 7.4) and fixed with a $95 \%$ ethanol solution. The nuclei were counterstained with 
DAPI (blue fluorescence). Next, the cells were visualized under fluorescence microscopy (Olympus, Japan).

\section{Western blot analysis}

AGS cells were cultured in a $25 \mathrm{~cm}$ flask. After reaching $80 \%$ confluence, the cells were treated with blank niosome (without drug), free DOX, Nio-DOX $\left(0.3 \mu \mathrm{g} \cdot \mathrm{mL}^{-1}\right)$, free QC, Nio-QC $\left(40 \mu \mathrm{g} \cdot \mathrm{mL}^{-1}\right)$, free siRNA, Nio-siRNA, Nioscrambled siRNA (100 nM), and Nio-DOX/siRNA for $72 \mathrm{hrs}$. Then, the cells were harvested and lysed in HES buffer. The protein concentration was quantified using the Bradford assay. Afterward, $50 \mu \mathrm{g}$ of samples were applied to a $10 \%$ SDS-PAGE and then transferred into a nitrocellulose membrane. To block non-specific binding, the membranes were incubated in 5\% BSA for $2 \mathrm{~h}$ at room temperature. Next, the membranes were incubated with CDC20 or $\beta$-actin primary antibody (1:1000 dilution) overnight at $4^{\circ} \mathrm{C}$. Blots were probed for $2 \mathrm{~h}$ at room temperature with a horseradish peroxidase-conjugated secondary antibody (1:2000). Finally, the visualization was performed with a chemiluminescence visualization system (Syngene GBOX Gel Documentation 680X) using ECL kit (GE Healthcare).

\section{Real-time quantitative PCR (RT-PCR)}

At first, AGS cells were seeded in 6-well plates, and then, treated with blank niosome, free DOX, Nio-DOX $(0.3 \mu \mathrm{g}$. $\left.\mathrm{mL}^{-1}\right)$, free $\mathrm{QC}$, Nio-QC (40 $\left.\mu \mathrm{g} \cdot \mathrm{mL}^{-1}\right)$, free siRNA, NiosiRNA, Nio-scrambled siRNA (100nM), and Nio-DOX/ siRNA for $72 \mathrm{hrs}$. The total RNA was isolated from the cultured AGS cells using Trizol reagent (Invitrogen, Carlsbad, CA) according to the manufacturer's instruction. After that, cDNA was synthesized using a Prime Script RT Master Mix kit (Takara, Dalian). Real-time PCR amplification was carried out using SYBR Premix Ex Taq II (Takara, Dalian) for 35 cycles using the following protocol: $95^{\circ} \mathrm{C}$ for $5 \mathrm{~min}, 95^{\circ} \mathrm{C}$ for $30 \mathrm{~s}, 60^{\circ} \mathrm{C}$ for $30 \mathrm{~s}, 72^{\circ} \mathrm{C}$ for $30 \mathrm{~s}$, and $72^{\circ} \mathrm{C}$ for $5 \mathrm{~min}$. The sequences of the primers were as follows: CDC20 sense 5'-GACCACTCCTAGCAAACCTGG-3' and antisense 5'-GGGCGTCTGGCTGTTTTCA-3', $\beta$-actin sense 5'-GATAGGGACATGCGGAGCCA-3', and antisense 5'CCAACCGCGAGAAGATGA-3'.

\section{Flow cytometry}

Cell apoptosis was detected using an Annexin V-FITC/ PI apoptosis analysis kit. AGS cells were seeded in 6well plates after reached $80 \%$ confluence and treated with free drugs, drugs-loaded nanoniosome, co-delivery of Nio-DOX + Nio-QC, CDC20siRNA/DOX-loaded niosome, and CDC20siRNA/DOX-loaded niosome + niosomal QC combination. The cells that did not receive any drug considered the control cells. After $72 \mathrm{hrs}$, the total cells were harvested by trypsinization, collected by centrifugation, washed with PBS, and suspended in $100 \mu \mathrm{L}$ binding buffer. Soon after, the cells were stained with Annexin V-FITC and propidium iodide (PI) and put in the dark place for $15 \mathrm{~min}$. The apoptotic cells were probed by a flow cytometer (BD Biosciences, USA).

\section{Results}

\section{Characterization of the nanoniosomal} formulation

\section{The effect of DOTAP molar ratio}

In the present study, we developed cationic PEGylated nanoniosomes that are able to protect siRNA against degradation by the nuclease accumulation in the tumor through the improved permeability and retention (EPR) effects. The above-mentioned niosomes also facilitate the cellular uptake by means of the electrostatic interactions with the membrane of the target cells in vitro. To evaluate whether the molar ratio of DOTAP would affect the particle size, PDI, the surface charge of nanoniosome for siRNA adsorption, the entrapment efficiency, the drug loading efficiency and the release, different amounts of DOTAP were added to create cationic PEGylated nanoniosome formulations. According to Table 1, niosome formulation (F5) containing 30\% DOTAP exhibited a smaller mean particle size and a higher drug entrapment efficiency. Zeta potential was increased by the addition of $30 \%$ DOTAP as a cationic lipid in the F5 $(+36.5 \mathrm{mV})$ as compared with the other formulations. On the other hand, the diameter of the vesicles was decreased in parallel with an increase in the molar ratios of DOTAP. As shown in Table 1, an increase in the concentration of DOTAP enhanced the drug-release rate which is attributed to the flexible structure of DOTAP and the drug leakage. The obtained findings showed that the niosome formulations containing Tween-60: cholesterol: DPPC: DOTAP: DSPEmPEG with molar ratios of 45: 5: 15: 30: 5 (F5) had the optimum feature in terms of small diameter, the number of positive charge vesicles, and high entrapment efficiency which were chosen for further analysis (Table 1). As shown in Table 2, the diameter of the nanoniosome lacking drug was $\sim 44.7 \mathrm{~nm}$, while the diameter for Nio-DOXsiRNA was increased to $\sim 62.7 \mathrm{~nm}$ due to the entrapment 
Table 2 Particle size, PDI and zeta potential of optimum formulation

\begin{tabular}{|l|l|l|l|}
\hline Optimum formulation & Particle size (nm) & Polydispersity index (PDI) & Zeta potential (mV) \\
\hline Blank niosome (no drugs) & $44.7 \pm 3.1$ & $0.16 \pm 0.02$ & $+41.2 \pm 1.7$ \\
Nio-DOX & $49.3 \pm 0.9$ & $0.22 \pm 0.05$ & $+36.5 \pm 2.1$ \\
Nio-QC & $47.2 \pm 1.9$ & $0.25 \pm 0.02$ & $+37.1 \pm 3.4$ \\
Nio-siRNA & $55.4 \pm 3.4$ & $0.19 \pm 0.05$ & $+32.8 \pm 2.5$ \\
Nio-DOX-siRNA & $62.7 \pm 2.6$ & $0.23 \pm 0.01$ & $+25.2 \pm 3.3$ \\
\hline
\end{tabular}

Abbreviations: Nio, niosomal; DOX, Doxorubicin; QC, Quercetin.

of the drug and adhesion of siRNA to nanoniosomes. Zeta potential of Nio-siRNA was approximately $+32.8 \mathrm{mV}$ that was decreased in comparison to nanoniosome lacking drug $(+41.2 \mathrm{mV})$ that confirmed the loading of siRNA on nanoniosomes.

\section{Morphological evaluation of the optimized formulation}

The particle size and morphology of blank niosome and Nio-DOX-siRNA were further characterized by AFM analysis (Figure 1). All findings were in good agreement with the results of DLS analysis. The morphological shapes of the nanoniosomal vesicles are depicted in Figure 2. The image clearly shows the bilayer structure of the nanovesicles and their hydrophilic and hydrophobic sections. The micrograph reveals the spherical shape and smooth surface of the nanoniosomes with narrow size distribution. No aggregates were observed in the microscopic studies.

\section{The thermosensitivity of the niosomes}

Differential Scanning Calorimetry was carried out to determine the phase transition temperature $(\mathrm{Tm})$ of cationic PEGylated niosome. As shown in Figure 3, cationic PEGylated niosome composed of Tween-60: cholesterol: DPPC: DOTAP: DSPE-mPEG at a molar ratio 45:5:15:30:5 had a $\mathrm{Tm}$ of $42.47^{\circ} \mathrm{C}$ that showed the thermosensitive nature of the cationic PEGylated niosomes.

\section{In vitro drug-release assay}

DOX and QC release behaviors were studied in release medium in $\mathrm{pH} 7.4$ at $37^{\circ} \mathrm{C}$ and $42^{\circ} \mathrm{C}$ (Figure 4) to mimic the temperature of healthy tissues and tumor microenvironment, respectively. Notably, a higher temperature of tumor microenvironment results from the increased metabolism of cancer cells in comparison to normal cells. During the release period, DOX and QC were sustainably released from cationic PEGylated nanoniosomes at a release rate of $31 \%$ and $47 \%$, respectively, at $37^{\circ} \mathrm{C}$ after
A

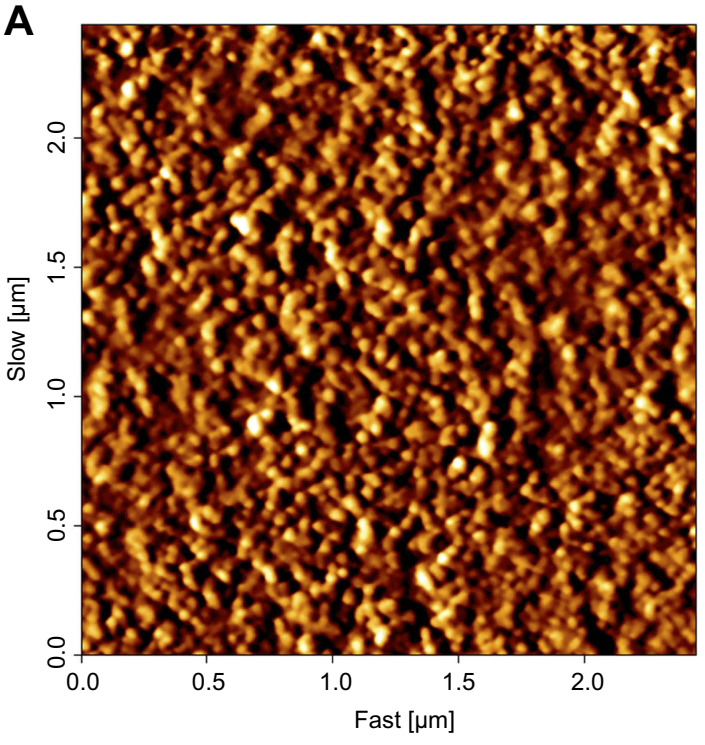

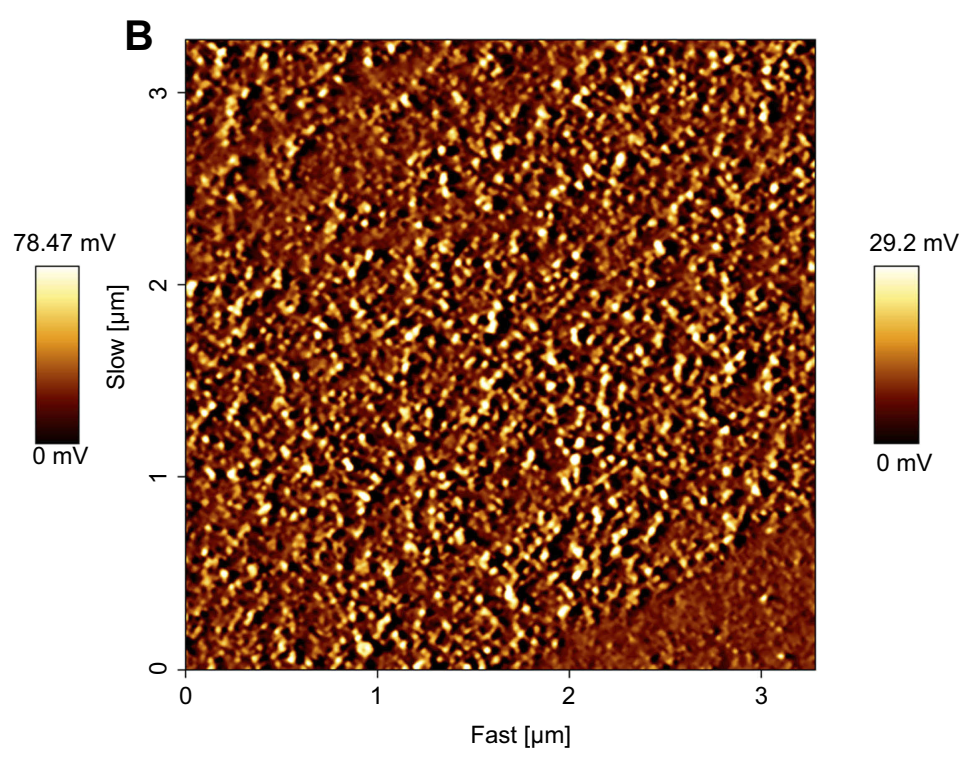

Figure I AFM images of blank niosome (A) and Nio-DOX-siRNA (B).

Abbreviations: AFM, atomic force microscope; Nio, niosomal; DOX, doxorubicin. 


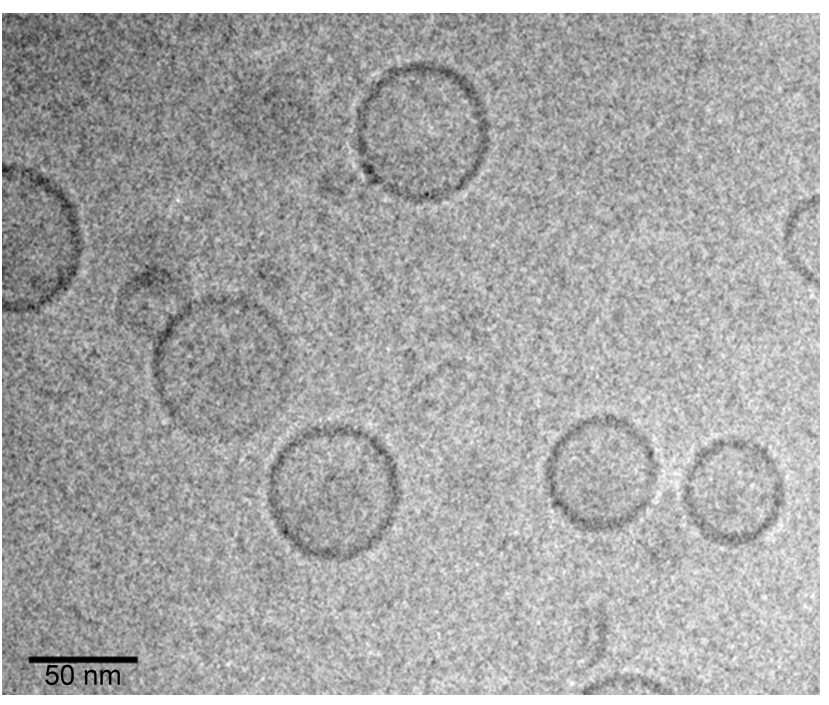

Figure 2 Cryo-TEM micrographs of optimum formulation of Nio-DOX-siRNA. Abbreviations: Cryo-TEM, Cryo-transmission electron microscopy; Nio, niosomal; DOX, doxorubicin.

$96 \mathrm{hrs}$. The release rate of DOX and QC was over 55\% and $68 \%$, respectively, at $42^{\circ} \mathrm{C}$, which was faster than that of $37^{\circ} \mathrm{C}$, indicating that the release rate of DOX and QC was enhanced under the higher temperature environment and the release pattern is suitable for tumor tissues.

\section{Cellular uptake assay}

In order to evaluate the cellular uptake behavior of various DOX/QC/FAM-labeled siRNA niosomal formulations in gastric cancer cells, the cellular uptake experiments were executed. Figure 5 demonstrates and compares the cellular uptake of the F2 (10\% DOTAP) and F5 (30\% DOTAP)

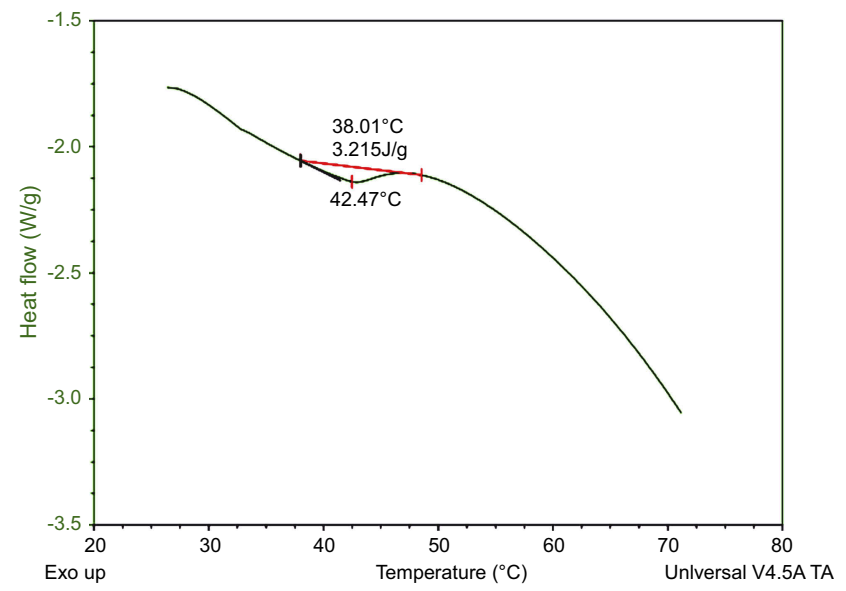

Figure 3 Differential scanning calorimetric scan (DSC) analysis of nanoniosome (Tween-60: cholesterol: DPPC: DOTAP: DSPE-mPEG 45:5:1 5:30:5) (molar ratio). Abbreviations: DPPC; I, 2-dipalmitoyl-sn-glycero-3-phosphocholin; DOTAP, I, 2dioleoyl-3-trimethylammonium propane; DSPE-mPEG; distearoylphosphoethanolamine-polyethylene glycol.
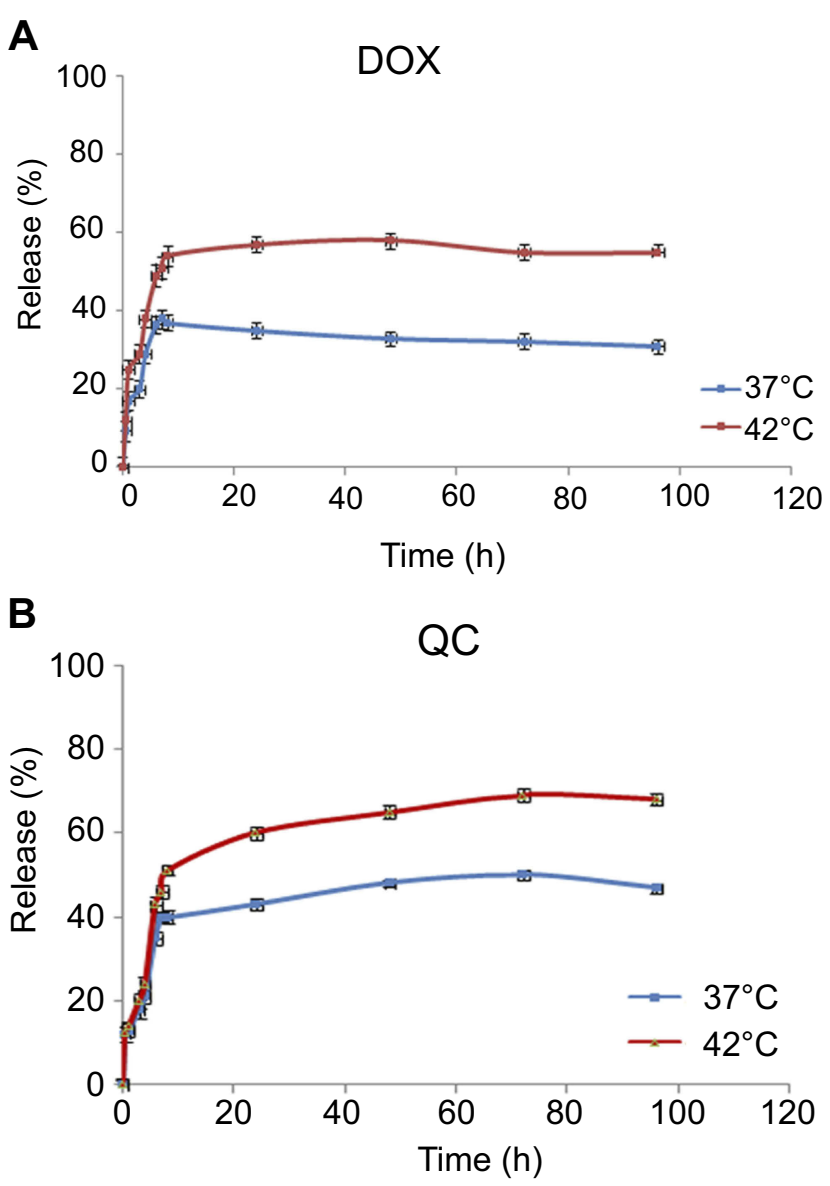

Figure 4 In vitro release profile of DOX (A) and QC (B) from niosomes after $72 \mathrm{hrs}$. Note: Data are represented as mean \pm SD $(n=3)$.

Abbreviations: DOX, doxorubicin; QC, quercetin.

formulations containing DOX/QC/FAM-labeled siRNA by AGS cell lines which was imaged by a fluorescence microscope. As illustrated in Figure 5A, the cells treated with niosomes-loaded DOX containing 30\% DOTAP showed a greater purple and turquoise blue color intensity, whereas in Figure 5B and C, QC and FAM-labeled siRNA niosomal formulations containing 30\% DOTAP indicated a higher green and cyan (blue-green) color intensity compared with the cells treated with 10\% DOTAP. These results demonstrated that the transfection efficiency of niosomal formulations was enhanced as the amount of DOTAP was heightened which was accompanied by the enhancement of the pharmacological effects for anticancer drugs.

\section{In vitro gene silencing studies}

Because cancer cells express a higher amount of CDC20 in comparison to the normal cells, it would be worth studying the efficacy of the formulated drugs on the 


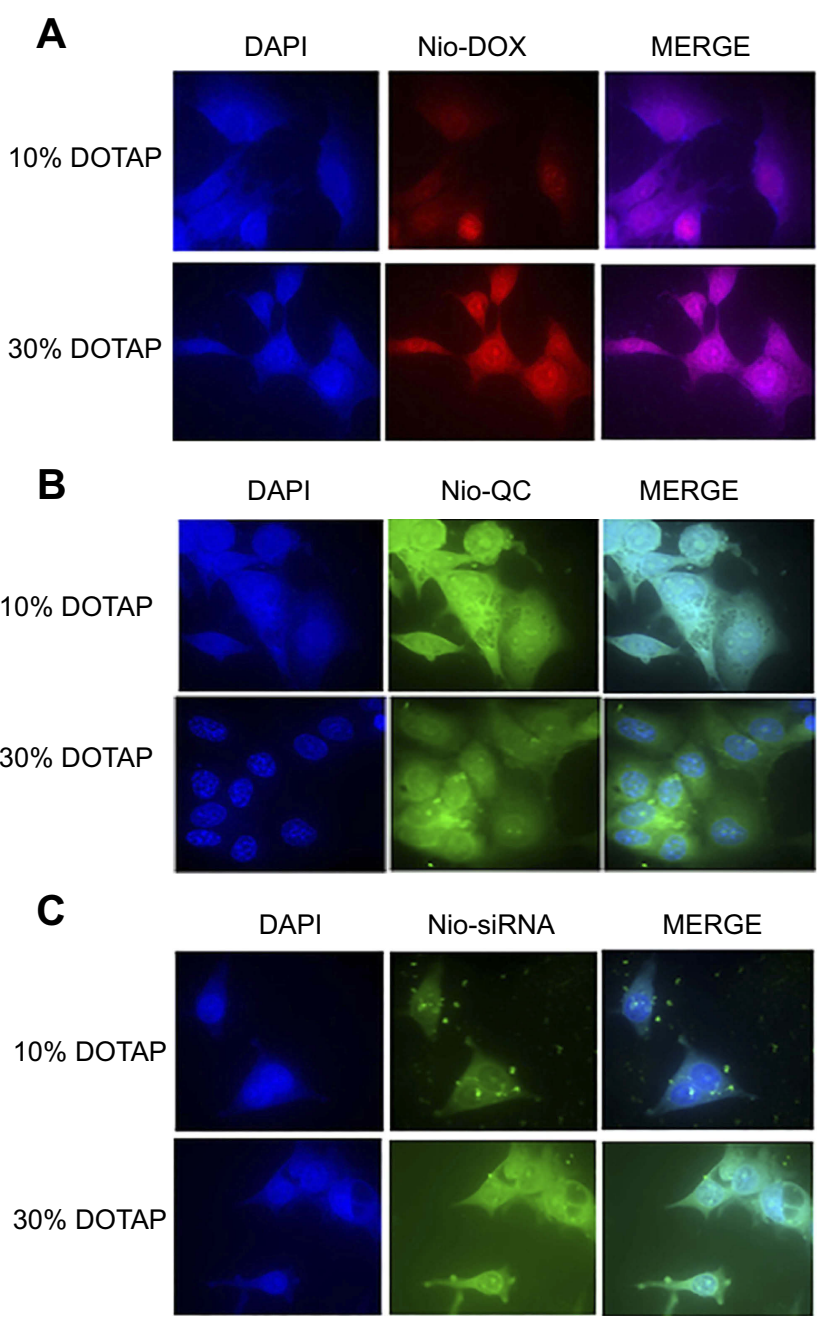

Figure 5 Cellular uptake of F2 (10\% DOTAP) and F5 (30\% DOTAP) DOX, QC and FAM-labeled siRNA-loaded nanoniosome formulations on AGS cell line. (A) NioDOX (B) Nio-QC (C) Nio-FAM-labeled siRNA (60x magnification).

Abbreviations: DOTAP, I, 2-dioleoyl-3-trimethylammonium-propane; Nio, niosomal; DOX, doxorubicin; QC, quercetin; DAPI, 4', 6-diamidino-2-phenylindole.

alteration of this particular gene to monitor and compare the potency of the designed niosomes. Hence, Western blot analysis and Real-time PCR were performed to measure the expression of CDC20 at the levels of protein and gene, respectively. The expression of $\mathrm{CDC} 20$ was analyzed to determine whether siRNA, DOX, and QC could decrease the level of CDC20. According to Figure 6, we found that $100 \mathrm{nM}$ CDC20siRNA remarkably reduced the expression of CDC20 at the protein level in AGS cells after $72 \mathrm{hrs}$ when compared to that in the untreated controls. Furthermore, increasing the concentration of CDC20siRNA (300 $\mathrm{nM})$ significantly decreased the expression of $\mathrm{CDC} 20$ at the protein level. Also, free siRNA could not dramatically reduce the level of CDC20 when compared with siRNA-containing niosome. We

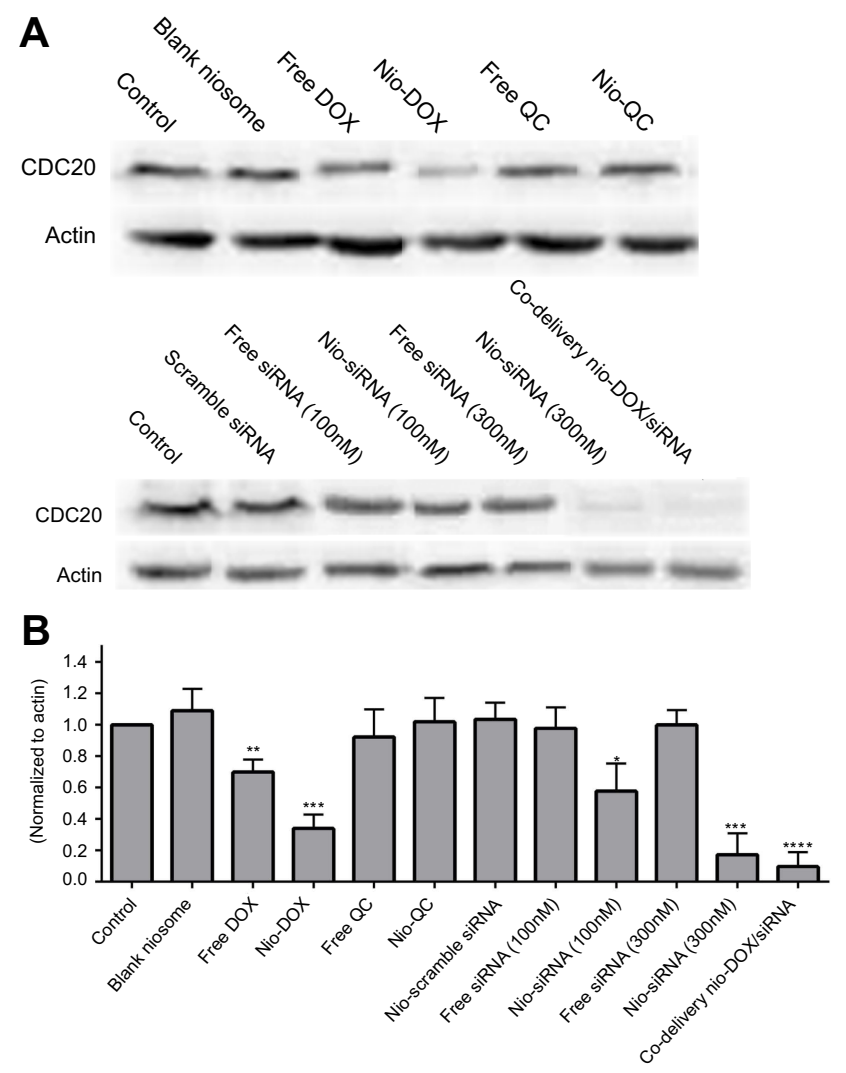

Figure 6 Silencing of CDC20 expression by different niosomes. (A) Western blot analysis of $C D C 20$ protein level. (B) Relative values for $C D C 20$ vs actin in A. Abbreviations: CDC20, cell division cycle 20; Nio, niosomal; DOX, doxorubicin; QC, quercetin.

observed that the cells incubated with the blank niosome and scramble siRNA showed the same expression level for CDC20 compared with the control. Correspondingly, codelivery of Nio-DOX/CDC20siRNA led to reduced CDC20 protein expression with a higher degree compared with the single- and free-form use of DOX or siRNA transfection. Contrarily, no significant difference was displayed among untreated cells, cells treated with free QC, and niosome-loaded QC. Intriguingly, these findings revealed that Nio-CDC20siRNA and Nio-DOX markedly reduced the gene expression of $\mathrm{CDC} 20$ as compared to the free forms. It should be noted, the results of Western blot analysis were in agreement with the results pertained to RT-PCR (Figure 7).

\section{Apoptosis analysis}

In our previous study, we assayed the cytotoxicity of the drugs and siRNA delivery system using the MTT method. Herein, we assessed the cytotoxicity of DOX, QC, and siRNA alone or in combination with each other by an alternative method performed by Annexin V-FITC/PI 


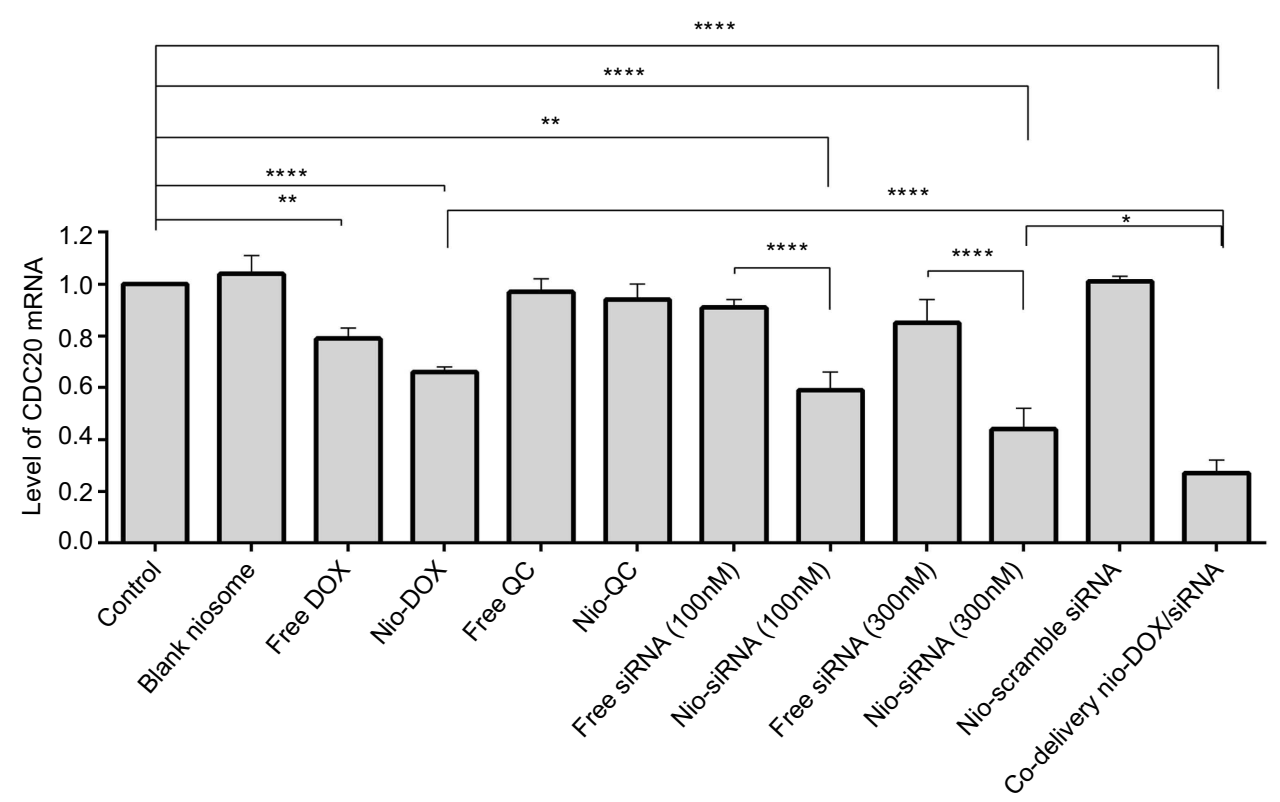

Figure 7 Real-time PCR analysis of CDC20 mRNA level in AGS cells transfected by different niosomes. The values were expressed as mean \pm SD from three independent experiments. ${ }^{*} p<0.05,{ }^{*} p<0.01,{ }^{* * *} p<0.001$, ${ }^{* * * *} p<0.000$ I. Abbreviations: CDC20, cell division cycle 20; Nio, niosomal; DOX, doxorubicin; QC, quercetin.

double staining. The cells either stained with Annexin VFITC or PI are individually probed by flow cytometry analysis. The results in Figure 8 indicate the apoptotic rate of AGS cells. The quadrant regions in flow cytometry chart, coined as Q1, Q2, Q3, and Q4, represent the living cells (double negative or FITC-/PI-), late apoptotic cells (double positive or $\mathrm{FITC}+\mathrm{PI}+$ ), early apoptotic cells (FITC+/PI-), and necrotic cells (FITC-/PI+), respectively. The apoptosis rate was $6.05 \%, 5.5 \%$, and $5.53 \%$ in cells treated with the free DOX, QC, and siRNA, respectively. However, the rate of apoptosis was increased to $18.1 \%$, $11.4 \%$, and $10.6 \%$ when the cells treated with niosomeloaded DOX, QC, and siRNA, respectively. Furthermore, there was a significant increase in the rate of cell death when DOX and siRNA were co-delivered resulted in a synergistic effect for the induction of apoptosis. The apoptosis rate was $31.2 \%$ in cells treated with co-delivery of Nio-DOX-siRNA in combination with Nio-QC which was recorded as the highest cell death rate in all groups $(p<0.05)$. These results are consistent with the cytotoxicity assay (MTT method) performed in our previous study.

\section{Discussion}

Chemotherapy is known as the most important treatment option for the management of gastric cancer. However, chemoresistance is one of the major obstacles that patients with gastric cancer should tackle it. Thus, preparing a multi-agents carrier system that can simultaneously deliver therapeutic siRNA and drugs into tumors cells might be considered a suitable candidate for the treatment of malignancies. In line with this, we developed a niosomal siRNA/drug delivery system that was able to carry a combination of chemical compounds, as well as siRNA against tumors to reduce the required doses of the drugs and the common side effects. The designed niosomes showed thermosensitivity, controlled drug release, improved passive targeting, drug stability, prolonged the circulation time, and increased drug concentration in tumor sites. Nanoniosomes have superiority to other kinds of drug delivery systems due to biocompatibilities, biodegradation, no allergic and toxic reactions, and low economic cost. ${ }^{11,12}$ In our previous study, we successfully prepared and optimized a cationic PEGylated niosome containing siRNA and drugs to combat the drug resistance and decrease the adverse effects. ${ }^{10}$ Recently, we evaluated and compared the effects of this multifunctional delivery system on the gene expression and apoptosis for the free and single usage of the components. In order to optimize nanoniosome for improving the loaded siRNA, we evaluated the different molar ratio of DOTAP. Also, cationic lipids supplemented with the niosomal formulations improved the physicochemical properties of niosomes and the transfection efficiency. The addition of DOTAP caused a decrease in the vesicle size and polydispersity index, which is associated with the further reciprocal repel force existing between the particles with the same charge in suspension system. ${ }^{13-15}$ To 

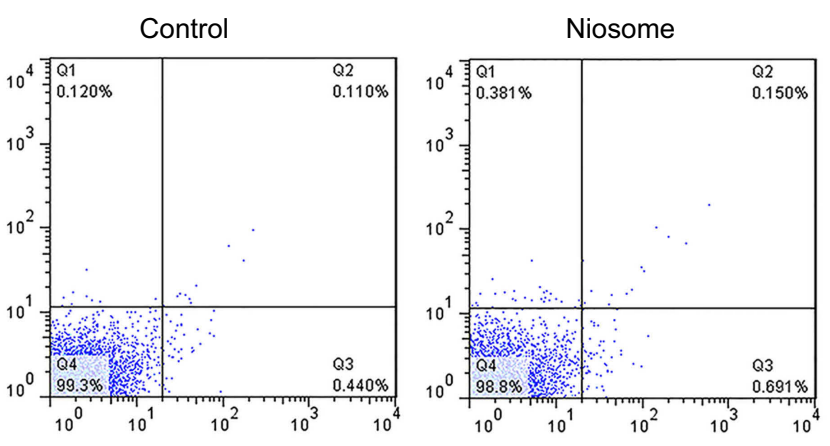

Free QC

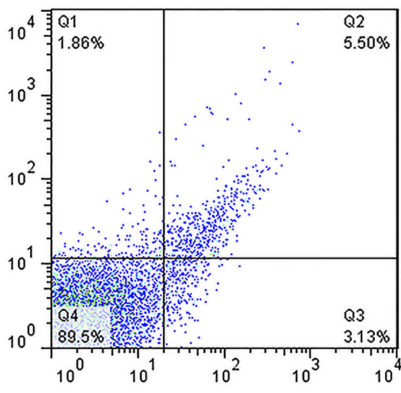

Nio-scramble-siRNA

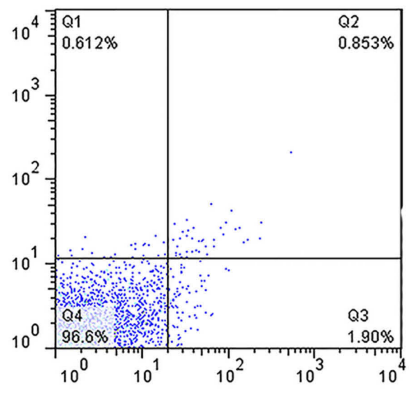

Nio-QC

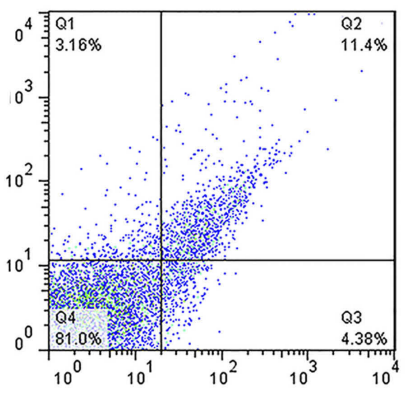

Nio-DOX-QC

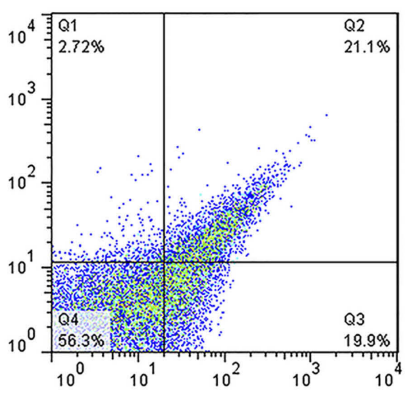

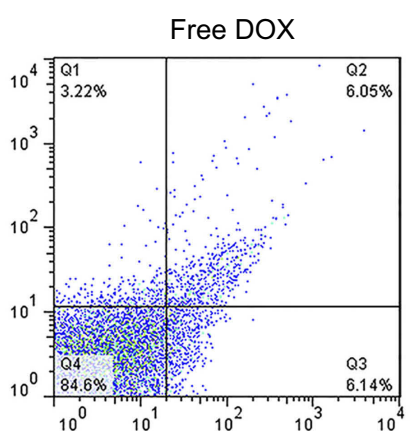

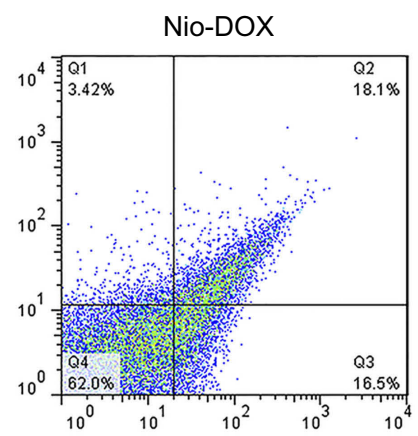

Free siRNA

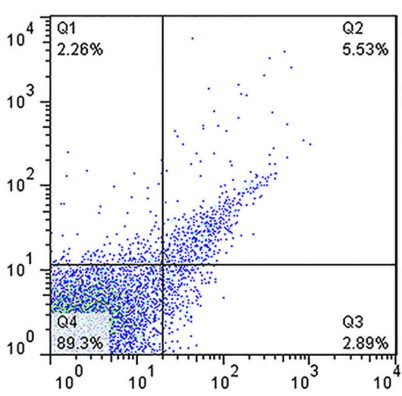

Co delivery Nio-DOX-siRNA

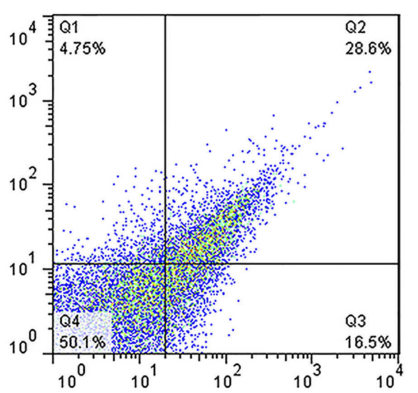

Nio-CDC20-siRNA

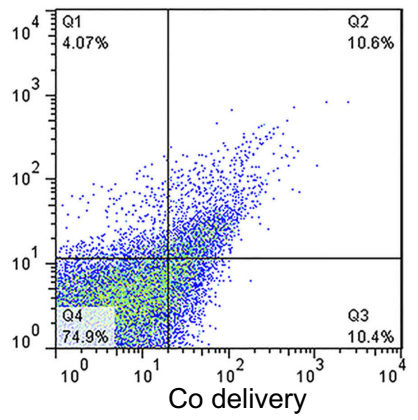

Nio-DOX-siRNA + Nio-QC

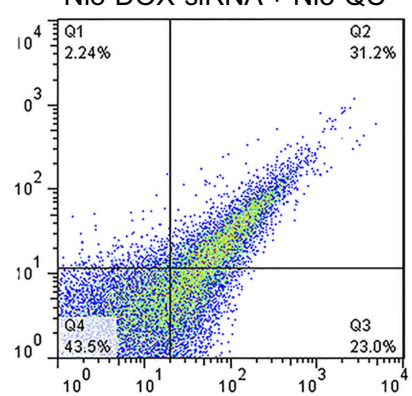

Figure 8 Cell apoptosis analyzed with Annexin V-FITC kit following the treatment of cells for 72 hrs. Abbreviations: Nio, niosomal; DOX, doxorubicin; QC, quercetin; FITC, fluorescein isothiocyanate.

hinder the aggregation of vesicular systems, it would be necessary to introduce a charge on the surface of the vesicle. A well-accepted indicator for the size of this barrier is zeta potential. If all the particles have large enough zeta potential, they probably repel each other enough that they will not have the propensity to aggregate. ${ }^{16}$ The cellular uptake assay was shown by the addition of DOTAP, which promoted the transfection efficiency of the DOX/QC/siRNA (F5) formula. It has been implicated that cationic lipids boost the transfection efficiency of the niosomal formulations. ${ }^{13,14}$ Our systems were designed as thermosensitive niosomes that such thermosensitivity was originated from the addition of dipalmitoyl phosphocholine (DPPC) to the membranes undergoing a gel to liquid crystalline phase transition at $\sim 42^{\circ} \mathrm{C}$. In this temperature, the hyperthermia occurs which, in turn, triggers the drug release and improves the passive targeting which eventually diminishes the adverse effect on the normal cells. ${ }^{17}$ For the regulation of normal mitosis and mitotic exit, the degradation of cyclin B1 by APC/C is needed. Basically, CDC20 acts as a key regulator of cancer development using the activation of APC which is responsible for the ubiquitin ligase activity. ${ }^{18,19}$ The knock-down of CDC20 expression at the transcriptional and translational levels was the main purpose of the present study. The overexpression of CDC20 in various tumors has been associated with invasion, proliferation, and metastasis of cancer cells. $5,7,20,21$ Silencing the expression of CDC20 by small interfering RNA (siRNA) inhibits the growth of solid tumor of melanoma, pancreatic carcinoma, and prostate cancer. $^{18,20}$ Thus, the effective delivery of siRNA to quench the expression of $\mathrm{CDC} 20$ as a potential target for cancer treatment is of great importance in the treatment of malignant tumors. DOX, as a common drug used in chemotherapy, 
impedes the growth of cancer cells and induces apoptosis. Nonetheless, myelosuppression, cardiotoxicity, the premature inactivation and the elimination of the normal cells can limit its therapeutic effects. ${ }^{22,23}$ Consequently, the use of the single and free DOX in the eradication of cancer cells is ineffective. Plant-derived chemical compounds have anti-cancer properties and are widely applied in the treatment of various cancers. QC is a plant flavonol found in many fruits, vegetables, and leaves which possess abroad spectrum of pharmacological effects. It is still unclear the chemosensitizer impact of QC on cancer cells when co-administrated with the chemotherapeutic agents. A study by Ramasamy and co-workers on the co-delivery of DOX and QC by $\mathrm{pH}$-sensitive polypeptidebased nanocarriers revealed that $\mathrm{QC}$ was able to enhance the cytotoxic effect of DOX significantly and also it induced marked cell apoptosis. These findings are consistent with the results obtained in the present study. ${ }^{24}$ Majumder et al demonstrated inhibition of CDC20 gene in the melanoma cells by a liposomal formulation of CDC20siRNA. They found out that CDC20siRNA was effective in silencing the expression of the CDC20 gene in the endothelial and tumor cells both at the mRNA and protein levels under the in vitro settings. These results are in agreement with the findings of our studies reported here. ${ }^{18}$ Bhunia and colleagues, exhibited the synergistic effect of CDC20siRNA and paclitaxel, employing nanometric liposomes, which inhibited xenografted neuroblastoma. ${ }^{25}$ The synergistic effects of QC and DOX on the gene expression of $\mathrm{CDC} 20$ have not been fully elucidated on the gastric cancer cells. This is the first study on the concomitant effect of CDC20siRNA, QC and DOX on the suppression of the $\mathrm{CDC} 20$ protein in the gastric cancer to the best of our knowledge. Results of the present research indicated highest level of inhibition of the growth of gastric cancer cells. In this study, the application of cationic PEGylated niosome, DOX, QC, and CDC20siRNA could be targeted to the tumor site under thermosensitive release. In the present study, at the cellular level, a proper inhibition of CDC20 expression has been achieved at both the transcriptional and translational levels by CDC20siRNA, respectively. It has been implied that the specific knockdown of CDC20 expression can suppress the cell growth of gastric cancer cells. The Codelivery Nio-DOX/CDC20siRNA exhibited the highest efficiency in silencing of $\mathrm{CDC} 20$ expression confirming that cationic PEGylated niosomes have high performance for the delivery of siRNA and the drugs. In detail, DOX is released from nanoniosomes and intercalated into DNA which results in the induction of DNA damage and activation of p53 gene, subsequently leading to the suppression of the CDC20 expression. ${ }^{26,27}$ The flow cytometry analysis represents an increase in cellular apoptosis rate in AGS cells when DOX and QC were encapsulated in nanoniosome formulations as compared to the free forms of the drugs $(p<0.05)$. Likewise, the apoptosis assay showed that the co-delivery Nio-DOX/ CDC20siRNA with Nio-QC was shown to have the highest apoptosis rate in gastric cancer cells compared with the free or single forms of the compounds. This phenomenon might be correlated to the higher cytotoxicity of the triple combination therapy in gastric cells. Our study showed that QC did not affect the silencing expression of CDC20, but it caused an induction in apoptosis rate and synergistically enhanced the toxicity effects of DOX. In conclusion, our results exhibited the controlled-release and synergistic anti-tumor effects of siRNA/anticancer drugs-containing nanoniosomes, which can downregulate the CDC20 expression in high efficiency resulting in synergistic therapeutic response for the elimination of human gastric cancer.

\section{Conclusion}

Our results revealed that the newly synthesized drug and gene co-delivery system represent combined characteristics of thermosensitive controlled release and synergistic anti-cancer potentials in vitro. The particle size and zeta potentials of this designed co-delivery system were suitable to be used for the intratumoral accumulation as it possessed high cellular uptake due to the electrostatic interactions with the cell membrane. DOX/CDC20siRNA provided a new platform for combination therapy to induce apoptosis and decrease the expression of the CDC20 protein. Therefore, this thermosensitive co-delivery system holds a great promise as a therapy option for the treatment of gastric cancer.

\section{Disclosure}

The authors report no conflicts of interest in this work.

\section{References}

1. Sitarz R, Skierucha M, Mielko J, Offerhaus GJA, Maciejewski R, Polkowski WP. Gastric cancer: epidemiology, prevention, classification, and treatment. Cancer Manag Res. 2018;10:239-48. doi:10.2147/ CMAR.S149619

2. Ramasamy T, Ruttala HB, Gupta B, et al. Smart chemistry-based nanosized drug delivery systems for systemic applications: a comprehensive review. J Control Release. 2017;258:226-253. doi:10.1016/j. jconrel.2017.04.043

3. Clavreul A, Pourbaghi-Masouleh M, Roger E, Menei P. Nanocarriers and nonviral methods for delivering antiangiogenic factors for glioblastoma therapy: the story so far. Int J Nanomedicine. 2019;14:2497513. doi:10.2147/IJN.S194858 
4. Wang L, Zhang J, Wan L, Zhou X, Wang Z, Wei W. Targeting Cdc20 as a novel cancer therapeutic strategy. Pharmacol Ther. 2015;151:141-151. doi:10.1016/j.pharmthera.2015.04.002

5. Wu W-J, Hu K-S, Wang D-S, et al. CDC20 overexpression predicts a poor prognosis for patients with colorectal cancer. J Transl Med. 2013;11(1):142. doi:10.1186/1479-5876-11-142

6. Chang DZ, Ma Y, Ji B, et al. Increased CDC20 expression is associated with pancreatic ductal adenocarcinoma differentiation and progression. J Hematol Oncol. 2012;5:15. doi:10.1186/1756-87225-15

7. Kato T, Daigo Y, Aragaki M, Ishikawa K, Sato M, Kaji M. Overexpression of CDC20 predicts poor prognosis in primary nonsmall cell lung cancer patients. J Surg Oncol. 2012;106(4):423-430. doi:10.1002/jso.23109

8. Ding ZY, Wu HR, Zhang JM, Huang GR, Ji DD. Expression characteristics of CDC20 in gastric cancer and its correlation with poor prognosis. Int J Clin Exp Pathol. 2014;7(2):722-727.

9. Wang G, Zhang J, Liu L, Sharma S, Dong Q. Quercetin potentiates doxorubicin mediated antitumor effects against liver cancer through p53/Bcl-xl. PLoS One. 2012;7(12):e51764. doi:10.1371/journal. pone.0051764

10. Hemati M, Haghiralsadat F, Yazdian F, Jafari F, Moradi A, Malekpour-Dehkordi Z. Development and characterization of a novel cationic PEGylated niosome-encapsulated forms of doxorubicin, quercetin and siRNA for the treatment of cancer by using combination therapy. Artif Cell Nanomed Biotechnol. 2019;47 (1):1295-1311. doi:10.1080/21691401.2018.1489271

11. Ag Seleci D, Seleci M, Walter J-G, Stahl F, Scheper T. Niosomes as Nanoparticular Drug Carriers: fundamentals and Recent Applications. J Nanomater. 2016;2016:13. doi:10.1155/2016/7372306

12. De Silva L, Fu J-Y, Htar TT, et al. Characterization, optimization, and in vitro evaluation of Technetium-99m-labeled niosomes. Int $J$ Nanomedicine. 2019;14:1101-1117. doi:10.2147/IJN.S184912

13. Ojeda E, Puras G, Agirre M, et al. The role of helper lipids in the intracellular disposition and transfection efficiency of niosome formulations for gene delivery to retinal pigment epithelial cells. Int $J$ Pharm. 2016;503(1-2):115-126. doi:10.1016/j.ijpharm.2016.02.043

14. Zhi D, Zhang S, Wang B, Zhao Y, Yang B, Yu S. Transfection efficiency of cationic lipids with different hydrophobic domains in gene delivery. Bioconjug Chem. 2010;21(4):563-577. doi:10.1021/ bc900393r

15. Ojeda E, Puras G, Agirre M, et al. Niosomes based on synthetic cationic lipids for gene delivery: the influence of polar head-groups on the transfection efficiency in HEK-293, ARPE-19 and MSC-D1 cells. Org Biomol Chem. 2015;13(4):1068-1081. doi:10.1039/ c4ob02087a
16. Ertekin ZC, Bayindir ZS, Yuksel N. Stability studies on piroxicam encapsulated niosomes. Curr Drug Deliv. 2015;12(2):192-199.

17. Chen J, Cheng D, Li J, et al. Influence of lipid composition on the phase transition temperature of liposomes composed of both DPPC and HSPC. Drug Dev Ind Pharm. 2013;39(2):197-204. doi:10.3109/ 03639045.2012 .668912

18. Majumder P, Bhunia S, Bhattacharyya J, Chaudhuri A. Inhibiting tumor growth by targeting liposomally encapsulated CDC20siRNA to tumor vasculature: therapeutic RNA interference. $J$ Control Release. 2014;180:100-108. doi:10.1016/j.jconrel.2014.02.012

19. Musacchio A, Salmon ED. The spindle-assembly checkpoint in space and time. Nat Rev Mol Cell Biol. 2007;8:379. doi:10.1038/nrm2163

20. Li K, Mao Y, Lu L, et al. Silencing of CDC20 suppresses metastatic castration-resistant prostate cancer growth and enhances chemosensitivity to docetaxel. Int J Oncol. 2016;49(4):1679-1685. doi:10.3892/ ijo.2016.3671

21. Choi J-W, Kim Y, Lee J-H, Kim Y-S. High expression of spindle assembly checkpoint proteins CDC20 and MAD2 is associated with poor prognosis in urothelial bladder cancer. Virchows Archiv. 2013;463(5):681-687. doi:10.1007/s00428-013-1473-6

22. Ramasamy T, Kim JH, Choi JY, et al. pH sensitive polyelectrolyte complex micelles for highly effective combination chemotherapy. $J$ Mate Chem B. 2014;2(37):6324-6333. doi:10.1039/C4TB00867G

23. Deng G, Chen $\mathrm{C}$, Zhang J, et al. Se@SiO2 nanocomposites attenuate doxorubicin-induced cardiotoxicity through combatting oxidative damage. Artif Cell Nanomed Biotechnol. 2018;46(supp2):112-121. doi:10.1080/21691401.2018.1452250

24. Ramasamy T, Ruttala H, Chitrapriya N, et al. Engineering of Cell Microenvironment-responsive Polypeptide Nanovehicle Co-encapsulating a Synergistic Combination of Small Molecules for Effective Chemotherapy in Solid Tumors. Acta Biomater. 2017;48:131-143. doi: 10.1016/j.actbio.2016.10.034

25. Bhunia S, Radha V, Chaudhuri A. CDC20siRNA and paclitaxel coloaded nanometric liposomes of a nipecotic acid-derived cationic amphiphile inhibit xenografted neuroblastoma. Nanoscale. 2017;9 (3):1201-1212. doi:10.1039/c6nr07532k

26. Vergoni B, Cornejo PJ, Gilleron J, et al. dna damage and the activation of the p53 pathway mediate alterations in metabolic and secretory functions of adipocytes. Diabetes. 2016;65(10):3062-3074. doi: $10.2337 / \mathrm{db} 16-0014$

27. Kidokoro T, Tanikawa C, Furukawa Y, Katagiri T, Nakamura Y, Matsuda K. CDC20, a potential cancer therapeutic target, is negatively regulated by p53. Oncogene. 2008;27(11):1562-1571. doi:10.1038/sj. onc. 1210799
International Journal of Nanomedicine

\section{Publish your work in this journal}

The International Journal of Nanomedicine is an international, peerreviewed journal focusing on the application of nanotechnology in diagnostics, therapeutics, and drug delivery systems throughout the biomedical field. This journal is indexed on PubMed Central, MedLine, CAS, SciSearch ${ }^{\mathbb{R}}$, Current Contents ${ }^{\mathbb{B}} /$ Clinical Medicine,
Journal Citation Reports/Science Edition, EMBase, Scopus and the Elsevier Bibliographic databases. The manuscript management system is completely online and includes a very quick and fair peer-review system, which is all easy to use. Visit http://www.dovepress.com/ testimonials.php to read real quotes from published authors. 